\title{
Optimal Demand Bidding for Time-Shiftable Loads
}

\author{
Hamed Mohsenian-Rad, Senior Member, IEEE
}

\begin{abstract}
Time-shiftable loads have recently received an increasing attention due to their role in creating load flexibility and enhancing demand response and peak-load shaving programs. In this paper, we seek to answer the following question: How can a time-shiftable load, that itself may comprise of several smaller time-shiftable subloads, submit its demand bids to the day-ahead and real-time markets so as to minimize its energy procurement cost? Answering this question is challenging because of the intertemporal dependencies in choosing the demand bids for timeshiftable loads, and also due to the coupling between demand bid selection and time-shiftable load scheduling problems. Nevertheless, we answer the above question for different practical bidding scenarios and based on different statistical characteristics of practical market prices. In all cases, closed-form solutions are obtained for the optimal choices of the price and energy bids. The bidding performance is then evaluated in details by examining several case studies and analyzing actual market price data.
\end{abstract}

Keywords: Optimal price and energy bids, time-shiftable load, day-ahead market, real-time market, demand side management, multi-stage stochastic optimization, closed-form solutions.

\section{INTRODUCTION}

The deregulation of electricity markets has provided the load entities with new and major opportunities to procure their energy needs from diverse resources in a competitive market in order to lower their energy expenditure. In the United States, large consumers and load serving entities (LSEs) are already eligible to participate in both the forward markets and the realtime markets that are operated by several Independent System Operators (ISOs), such as the Electric Reliability Council of Texas (ERCOT) [1], the Pennsylvania-Jersey-Maryland (PJM) Interconnection [2], and the California ISO [3].

In a typical two-settlement electricity market, load entities can procure energy from a day-ahead market and a real-time market [4]. Power procurement is facilitated by submitting demand bids. The demand bids that are submitted to the dayahead market indicate an energy quantity and possibly also a price quantity. The bids that are submitted (or metered) to the real-time market only indicate an energy quantity [1]-[3].

In this paper, our focus is on finding optimal demand bids for time-shiftable loads, a.k.a. deferrable loads with deadlines. In brief, a time-shiftable load is a task that requires consuming a certain total energy to finish, but its operation can be scheduled any time within a given time frame, where the end of such time frame is the deadline to finish operation. Some examples of time-shiftable loads include: charging electric vehicles [5], [6], intelligent pools [7], irrigation pumps [8], water heaters [9], batch processes in data centers and computer servers [10], [11], industrial equipment in process control and manufacturing [12], [13], and various home appliances such

The author is with the Department of Electrical Engineering, University of California, Riverside, CA, USA, e-mail: hamed@ee.ucr.edu. This work was supported by NSF grants ECCS 1253516, ECCS 1307756, and CNS 1319798. as washing machine, dryer, and dish-washer [14]-[19]. Timeshiftable loads have recently received a great deal of attention due to their role in demand response and peak-load shaving programs. In many cases, a time-shiftable load consists of several smaller time-shiftable subloads or subtasks.

\section{A. Summary of Technical Contributions}

The immediate application of the designs in this paper is for large time-shiftable loads in different sectors (or their LSEs) to optimally bid in the electricity markets. But this work is also beneficial to demand response programs, as it can help utilities and aggregators to better exploit their time-shiftable load potentials to lower their total energy procurement cost.

The analysis in this paper is challenging, first and foremost, because of the inter-temporal dependencies in choosing the demand bids for time-shiftable loads. Furthermore, the bid selection problem for time-shiftable loads is inherently coupled with the problem of time-shiftable load scheduling. The contributions in this paper can be summarized as follows:

- A time-coupled multi-stage stochastic optimization problem is formulated for selecting price and energy bids to the day-ahead market and energy bids to the real-time market to operate time-shiftable loads with deadlines.

- Two design scenarios are considered: the case where persubload bidding is allowed; and the case where the timeshiftable load is mandated by the market to submit single demand bids, regardless of the number of its subloads.

- For each design, the impact and importance of holding or removing the assumption on price independence across the day-ahead and real-time markets are investigated.

- Several case studies are analyzed using PJM price data. The impact of different price scenarios, subload configurations and sizes, and bidding rules are investigated.

\section{B. Related Work}

The related work in the literature can be classified into three separate groups. One thread of research that has emerged only recently focuses on modeling and utilizing time-shiftable loads in the context of smart grid and demand side management. For example, the problem of scheduling time-shiftable loads under different retail electricity pricing scenarios is addressed in [16], [17], [19] for residential loads, in [12], [13] for industrial and commercial loads, and in [20] for electric vehicle charging stations. As another example, there are studies on optimizing the operation of time-shiftable loads for different design objectives such as peak-load shaving [18], voltage control or frequency regulation [21], and integration of renewable energy resources [22]. However, the prior studies do not address the issue of optimal demand bidding for time-shiftable loads. 
The second group of studies discuss the general problem of demand bidding in deregulated electricity markets, but they do not consider time-shiftable loads. For example, in [23], [24], the authors explained how an LSE should bid in the wholesale market based on some fixed or forecasted load data. In this regard, our paper can be seen as an extension of [23], [24] to the case of serving time-shiftable loads. The papers in this second group also include [25], [26], where the focus is on understanding the strategic interactions between bidding load entities and bidding generators using game theory.

Finally, there are some recent studies on linking demand response to market operations for price-elastic loads, where the load is modeled using a concave utility function. For example, the joint problem of power procurement and demand response for price-elastic loads was addressed in [27], [28], with and without the presence of renewable generators. Our work is different in two key aspects. First, the studies in [27], [28] do not - in any way - discuss the problem of selecting the demand bids and the uncertainties that arise on whether the bids will be cleared in the market. Second, since the focus in [27], [28] is on price-elastic power loads, they do not address the challenges with respect to inter-temporal dependencies that are caused by time-shiftable energy loads and their requirements on finishing certain jobs by certain deadlines. The papers in this group also include [29], where new complex bidding rules are proposed for demand response aggregators. In contrast, here, we obtain the optimal price and energy bids for timeshiftable loads based on the existing bidding structures.

\section{Problem Statement}

\section{A. Two-settlement Electricity Market}

Consider an organized wholesale electricity market, such as the ones that are operated by PJM, ERCOT, and California ISO in the United States [1]-[3]. Under a two-settlement market framework, energy trading can be done at both day-ahead markets and real-time markets. Load entities can participate in these markets by submitting demand bids. In general, a demand bid may or may not include a price component [4]. If a demand bid includes both energy and price components, then it indicates that the load is willing to purchase the given quantity of energy only if the price is equal to or below the price bid. Depending on the ISO, such demand bids are referred to as Limit Order bids [1], Decrement bids [2], or Economic bids [3]. If a demand bid includes only an energy component, but not a price component, it indicates that the load is willing to purchase the given quantity of energy, regardless of the price. Such demand bids are referred to as Fixed Demand bids [1] or Self-Schedule bids [3]. In this paper, our focus is on submitting demand bids with price components, i.e., of type Limit Order, to the day-ahead market, and bids without price component, i.e., of type Fixed Demand, to the real-time market.

\section{B. Time-Shiftable Load}

The time-shiftable load model in this paper is similar to those that are widely used in the demand response literature, e.g., in [12], [13], [16]-[22]. A time-shiftable load is assumed to comprise $L \geq 1$ subloads, where for each subload $l=$ $1, \ldots, L$, parameters $\alpha_{l}$ and $\beta_{l}$ indicate the beginning and the end of the time interval at which the operation of subload $l$ can be scheduled, where $\alpha_{l}<\beta_{l}$. A higher $\beta_{l}-\alpha_{l}$ indicates more time flexibility in scheduling the operation of subload $l$. Parameter $\beta_{l}$ gives the deadline to complete the operation of subload $l$. The operation of each subload $l$ requires procuring and consuming a total of $e_{l} \geq 0 \mathrm{MWh}$ of energy to complete.

\section{Optimal Demand Bidding Problem}

Let us divide the operation time into $T$ equal time-slots based on the market bidding intervals and the time-shiftable load scheduling horizon. For example, $T=24$ for a daily operation of a time-shiftable load over hourly market intervals. The optimal demand bidding problem for a time-shiftable load can be formulated as the following optimization problem:

$$
\begin{aligned}
\underset{\text { Bids }}{\operatorname{Min}} \sum_{t=1}^{T} \mathbb{E}\{\operatorname{Cost} \text { (Procured Energy } \\
\text { During Time Slot } t)\} \\
\text { S.t. } \quad \sum_{t=\alpha_{1}}^{\beta_{1}}\left[\text { Procured Energy for } 1^{\text {th }}\right. \text { Subload } \\
\text { During Time Slot } t]=e_{1}
\end{aligned}
$$

$$
\sum_{t=\alpha_{L}}^{\beta_{L}}\left[\text { Procured Energy for } L^{\text {th }}\right. \text { Subload }
$$

During Time Slot $t]=e_{L}$,

where $\mathbb{E}$ denotes the expected value. The above problem is a $T+1$ stage stochastic optimization problem [30]. At the first stage, the Limit Order bids are submitted to the day-ahead market. The other $T$ stages correspond to submitting $T$ Fixed Order bids to the $T$ bidding cycles of the real-time market.

The reason for problem (1) to be a stochastic optimization problem is the uncertainty in the electricity prices, both at the day-ahead market and at the real-time market. The variables are all demand bids, which comprise $T$ variables for the energy components of the Fixed demand bids in the real-time market and $2 T$ variables for the energy and price components for the Limit Order demand bids in the day-ahead market. We seek to choose the day-ahead and real-time demand bids so as to minimize the combined expected cost of procuring power across the two-settlement market, subject to completing the operation of all subloads before their deadlines. Throughout the paper, we assume that the time-shiftable load is price-taker.

\section{The Case with One Subload}

In this section, we address the special case with $L=1$, i.e., when the time-shiftable load comprises only one subload. The general case with $L>1$ will be discussed in Section IV.

Consider the $T+1$ stage bidding framework in Fig. 1. If $L=1$, then we can assume that $\alpha_{1}=1$ and $\beta_{1}=T$. Energy 


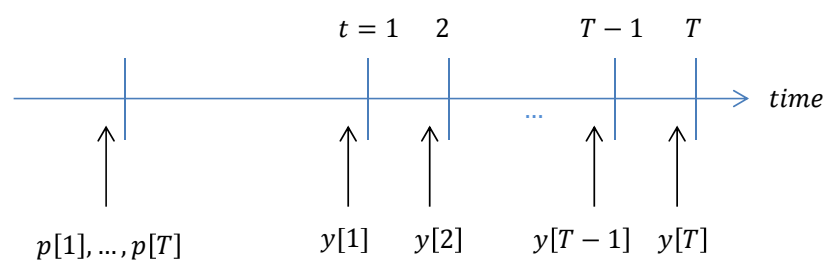

$x[1], \ldots, x[T]$

(Forward Market)

(Real - Time Market)

Fig. 1. The $T+1$ stage demand bidding framework for time-shiftable loads.

is procured from the day-ahead market by submitting Limit Order bids $\boldsymbol{p} \triangleq p[1], \ldots, p[T]$ and $\boldsymbol{x} \triangleq x[1], \ldots, x[T]$, where $\boldsymbol{p}$ is the vector of price bids and $\boldsymbol{x}$ is the vector of energy bids. Once the day-ahead market is settled and the clearing market prices $a[1], \ldots, a[T]$ are realized, if $a[t] \leq p[t]$, then the time-shiftable load procures the generation right of $x[t] \mathrm{MWh}$ during time slot $t$. If $a[t]>p[t]$, then no energy is procured from the day-ahead market for time slot $t$. As we approach the operation time, energy may be procured also from the realtime market by submitting (or metering) Fixed Demand bids $\boldsymbol{y} \triangleq y[1], \ldots, y[T]$. Under Fixed Demand bidding, procuring $y[t]$ MWh of energy is guaranteed, but the cost of such energy procurement is determined only after the real-time market is settled and the clearing market price $b[t]$ is realized [4].

\section{A. Selecting the Optimal Bids to Real-Time Market}

Assume that the bids $\boldsymbol{p}$ and $\boldsymbol{x}$ are submitted to the day-ahead market; the day-ahead market is settled; and the day-ahead prices are realized as $a[1], \ldots, a[T]$. The amount of energy that is procured from the day-ahead market is obtained as

$$
X=\sum_{t=1}^{T} \mathbb{I}(a[t] \leq p[t]) x[t],
$$

where $\mathbb{I}(\cdot)$ is the $0-1$ indicator function. If $a[t] \leq p[t]$, then $\mathbb{I}(a[t] \leq p[t])=1$; otherwise, $\mathbb{I}(a[t] \leq p[t])=0$. Note that, $0 \leq X \leq e_{1}$. Given $X$, and based on the bidding framework in Fig. 1, the bid $y[1]$ is submitted during the first cycle of the real-time market. Once the first cycle is settled, the price $b[1]$ is determined. Then, given $X, y[1]$, and $b[1]$, the bid $y[2]$ is submitted during the second cycle of the real-time market. These bidding cycles will continue until the last time slot where, given $X, y[1], \ldots, y[T-1]$, and $b[1], \ldots, b[T-1]$, the time-shiftable load submits the bid $y[T]$. At each bidding stage $t$, the goal is to minimize the expected value of the energy procurement cost in the remaining $T-t$ stages, while ensuring that the total energy $e$ will be procured by the time that we reach the load deadline at the end of time slot $T$.

Theorem 1: If the number of subloads is $L=1$, then, once the day-ahead market is cleared and $X$ in (2) is known, the optimal energy bids to the real-time market are obtained as

$$
y[t]=\mathbb{I}\left(t=t_{b}\right)\left(e_{1}-X\right), \quad t=1, \ldots, T,
$$

where

$$
t_{b}=\underset{1 \leq t \leq T}{\arg \min } \mathbb{E}\{b[t]\} .
$$

The proof of Theorem 1 is given in Appendix A. From Theorem 1, once the day-ahead market is cleared, the demand bidding process at the real-time market reduces to purchasing the entire remaining energy $e_{1}-X$ at time slot $t_{b}$, i.e., the time slot with the lowest real-time market expected price during time interval $[1, T]$. Given the realization of price $b\left[t_{b}\right]$, the cost of such real-time market purchase is $b\left[t_{b}\right]\left(e_{1}-X\right)$.

\section{B. Selecting the Optimal Bids to Day-Ahead Market}

Since submitting the bids to the day-ahead market is the first stage in the $T+1$ stage bidding process in Fig. 1, no prior knowledge is assumed to be available. However, the decision process must take into account the fact that once $\boldsymbol{p}$ and $\boldsymbol{x}$ are submitted and the day-ahead market is cleared, then the bids to the real-time market will be chosen as in (3). From (1), (2), and (3), we can formulate the stochastic optimization problem for selecting the bids to the day-ahead market as follows:

$$
\begin{aligned}
& \underset{\boldsymbol{p} \geq 0, \boldsymbol{x} \geq 0}{\operatorname{Min}_{t=1}} \mathbb{E}\left\{\sum_{t=1}^{T} \mathbb{I}(a[t] \leq p[t]) a[t] x[t]\right. \\
& \left.+b\left[t_{b}\right]\left(e_{1}-\sum_{t=1}^{T} \mathbb{I}(a[t] \leq p[t]) x[t]\right)\right\} \\
& \text { S.t. } \quad \sum_{t=1}^{T} \mathbb{I}(a[t] \leq p[t]) x[t] \leq e_{1}, \quad \forall a[1], \ldots, a[T],
\end{aligned}
$$

where the first and the second lines in the objective function denote the expected cost of energy procurement from the dayahead market and the real-time market, respectively. Here, the price values $a[1], \ldots, a[T]$ and $b\left[t_{b}\right]$ are random variables.

Since the inequality constraints in (5) must hold for any realization of the day-ahead market prices $a[1], \ldots, a[T]$, we need any binary-weighted summation of the day-ahead market energy bids $x[1], \ldots, x[T]$ to be less than or equal to $e_{1}$. Therefore, the feasible set that is constructed by the infinite number of constraints in (5) is equal to the feasible set that is constructed by a single constraint $\sum_{t=1}^{T} x[t] \leq e_{1}$. From this, and after removing the fixed term $\mathbb{E}\left\{b\left[t_{b}\right]\right\} e_{1}$ from the objective function in (5), we can rewrite problem (5) as

$$
\begin{array}{ll}
\underset{\boldsymbol{p} \geq 0, \boldsymbol{x} \geq 0}{\operatorname{Min}} & \sum_{t=1}^{T} \mathbb{E}\left\{\left(a[t]-b\left[t_{b}\right]\right) \mathbb{I}(a[t] \leq p[t])\right\} x[t] \\
\text { S.t. } & \sum_{t=1}^{T} x[t] \leq e_{1} .
\end{array}
$$

The difficulty in solving the above problem is the presence of the 0-1 indicator functions. Nevertheless, we can obtain closed-form solutions for problem (6) as we will show next.

1) Design I: Assuming Independent Markets: First, suppose that the day-ahead market prices $a[1], \ldots, a[T]$ are independent from the price $b\left[t_{b}\right]$ at time slot $t_{b}$ in the real-time market. In that case, the objective function in problem (6) becomes

$$
\sum_{t=1}^{T} \mathbb{E}\left\{\left(a[t]-\mathbb{E}\left\{b\left[t_{b}\right]\right\}\right) \mathbb{I}(a[t] \leq p[t])\right\} x[t],
$$

where the outmost expected value is now with respect to only $a[t]$. Therefore, we can show the following key theorem. 
Theorem 2: If the number of subloads is $L=1$ and under the market price independence assumption, the optimal price bids to the day-ahead market are obtained as

$$
p[1]=\ldots=p[T]=\mathbb{E}\left\{b\left[t_{b}\right]\right\} .
$$

The proof of Theorem 2 is given in Appendix B. From Theorem 2, at optimality, all price bids to day-ahead market are equal to the lowest expected price in the real-time market. From (8), the objective function in (6) further reduces to

$$
\sum_{t=1}^{T} \mathbb{E}\left\{\left(a[t]-\mathbb{E}\left\{b\left[t_{b}\right]\right\}\right) \mathbb{I}\left(a[t] \leq \mathbb{E}\left\{b\left[t_{b}\right]\right\}\right)\right\} x[t] .
$$

Since at each time slot $t$ and for any realization of $a[t]$, we have $\left(a[t]-\mathbb{E}\left\{b\left[t_{b}\right]\right\}\right) \mathbb{I}\left(a[t] \leq \mathbb{E}\left\{b\left[t_{b}\right]\right\}\right) \leq 0$, all the $T$ expected value terms in (9) are always non-positive. Therefore, the problem of choosing the energy bids $\boldsymbol{x}$ boils down to the following linear program with non-positive coefficients:

$$
\begin{array}{ll}
\underset{\boldsymbol{x} \geq 0}{\operatorname{Min}} & \sum_{t=1}^{T} \mathbb{E}\left\{\left(a[t]-\mathbb{E}\left\{b\left[t_{b}\right]\right\}\right) \mathbb{I}\left(a[t] \leq \mathbb{E}\left\{b\left[t_{b}\right]\right\}\right)\right\} x[t] \\
\text { S.t. } & \sum_{t=1}^{T} x[t] \leq e_{1} .
\end{array}
$$

The next theorem explains the closed-form solution of (10).

Theorem 3: The optimal energy bids to day-ahead market are

$$
x[t]=\mathbb{I}\left(t=t_{a}\right) e_{1}, \quad t=1, \ldots, T,
$$

where

$$
t_{a}=\underset{1 \leq t \leq T}{\arg \min } \mathbb{E}\left\{\left(a[t]-\mathbb{E}\left\{b\left[t_{b}\right]\right\}\right) \mathbb{I}\left(a[t] \leq \mathbb{E}\left\{b\left[t_{b}\right]\right\}\right)\right\} .
$$

The proof of Theorem 3 is given in Appendix C. From Theorem 3, if the time-shiftable load has $L=1$ subload, then it must submit only one non-zero energy bid to the day-ahead market. The timing of such non-zero bid is $t_{a}$ that is calculated as in (12). Since there is no reason to submit a non-zero price bid $p[t]$ if the energy bid $x[t]$ is zero; from the results in Theorems 2 and 3, we can rewrite the optimal price bids as

$$
p[t]=\mathbb{I}\left(t=t_{a}\right) \mathbb{E}\left\{b\left[t_{b}\right]\right\}, \quad t=1, \ldots, T .
$$

2) Design II: Not Assuming Independent Markets: Next, we relax the assumption about the independence of day-ahead and real-time market prices. Thus, we may no longer replace $b\left[t_{b}\right]$ in (6) with $\mathbb{E}\left\{b\left[t_{b}\right]\right\}$. Accordingly, we cannot use Theorem 2 anymore. Nevertheless, we can still find a closed-form solution for problem (6) through a slightly different analysis.

Suppose that the price bid vector $\boldsymbol{p}$ is already selected. Then, the problem of finding the energy bid vector $\boldsymbol{x}$ becomes

$$
\begin{array}{ll}
\underset{\boldsymbol{x} \geq 0}{\operatorname{Min}} & \sum_{t=1}^{T} \mathbb{E}\left\{\left(a[t]-b\left[t_{b}\right]\right) \mathbb{I}(a[t] \leq p[t])\right\} x[t] \\
\text { S.t. } & \sum_{t=1}^{T} x[t] \leq e_{1} .
\end{array}
$$

The above problem is a linear program, but its coefficients are not guaranteed to be non-positive. For notational simplicity, we denote the coefficient of variable $x[t]$ in (14) as

$$
\mathbb{C}(t, p[t])=\mathbb{E}\left\{\left(a[t]-b\left[t_{b}\right]\right) \mathbb{I}(a[t] \leq p[t])\right\} .
$$

The extension of Theorem 3 in this case is as follows.

Theorem 4: Given $\boldsymbol{p}$, the solution of problem (14) is

$$
x[t]=\mathbb{I}\left(t=t_{a, \boldsymbol{p}}\right) \mathbb{I}\left(\mathbb{C}\left(t_{a, \boldsymbol{p}}, p\left[t_{a, \boldsymbol{p}}\right]\right) \leq 0\right) e_{1}, \quad t=1, \ldots, T,
$$

where

$$
t_{a, \boldsymbol{p}}=\underset{1 \leq t \leq T}{\arg \min } \mathbb{C}(t, p[t]) .
$$

The proof of Theorem 4 is given in Appendix D. Note that, if $\mathbb{I}\left(\mathbb{C}\left(t_{a, \boldsymbol{p}}, p\left[t_{a, \boldsymbol{p}}\right]\right) \leq 0\right)=0$, then all the coefficients in the objective function in (14) are positive; and at optimality, we have $x[1]=\ldots=x[T]=0$. From Theorem 4 , for the given $\boldsymbol{p}$, the optimal objective value in (14) is obtained as

$$
\mathbb{I}\left(\mathbb{C}\left(t_{a, \boldsymbol{p}}, p\left[t_{a, \boldsymbol{p}}\right]\right) \leq 0\right) \mathbb{C}\left(t_{a, \boldsymbol{p}}, p\left[t_{a, \boldsymbol{p}}\right]\right) e_{1} .
$$

If $\mathbb{C}\left(t_{a, \boldsymbol{p}}, p\left[t_{a, \boldsymbol{p}}\right]\right) \leq 0$, then the optimal objective value in (18) is $\mathbb{C}\left(t_{a, \boldsymbol{p}}, p\left[t_{a, \boldsymbol{p}}\right]\right) e_{1}$; otherwise, the optimal objective value is zero. From (17) and (18), and since $e_{1}$ is a constant, the problem of finding the optimal price bid vector $\boldsymbol{p}$ in (14) boils down to solving the following optimization problem:

$$
\begin{aligned}
\underset{\boldsymbol{p} \geq 0}{\operatorname{Min}} \mathbb{I}\left(\mathbb{C}\left(t_{a, \boldsymbol{p}}, p\left[t_{a, \boldsymbol{p}}\right]\right) \leq 0\right) \mathbb{C}\left(t_{a, \boldsymbol{p}}, p\left[t_{a, \boldsymbol{p}}\right]\right) \\
=\operatorname{Min}_{\boldsymbol{p} \geq 0} \min _{1 \leq t \leq T} \mathbb{C}(t, p[t]) \mathbb{I}(\mathbb{C}(t, p[t]) \leq 0) \\
\quad=\min _{1 \leq t \leq T} \underset{p[t] \geq 0}{\operatorname{Min}} \mathbb{C}(t, p[t]) \mathbb{I}(\mathbb{C}(t, p[t]) \leq 0),
\end{aligned}
$$

where the first equality is the direct result of the definition of $t_{a, \boldsymbol{p}}$ in (17), and the second equality holds because $\mathbb{C}(t, p[t]) \mathbb{I}(\mathbb{C}(t, p[t]) \leq 0)$ depends only on $p[t]$ but not on $p[1], \ldots, p[t-1], p[t+1], \ldots, p[T]$. Here, we use the fact that

$$
\min _{v, w} \min \{f(v), g(w)\}=\min \left\{\min _{v} f(v), \min _{w} g(w)\right\} \text {. }
$$

From (19), finding the optimal price bid vector $\boldsymbol{p} \geq 0$ under Design II is decomposed into $T$ separate subproblems over time slots $t=1, \ldots, T$. For each time slot $t$, we choose the scalar price bid $p[t] \geq 0$ such that we minimize the corresponding coefficient term in (15), if it is non-positive.

Theorem 5: If the number of subloads is $L=1$ and we relax any market price dependency assumption, then the optimal price bids to the day-ahead market are obtained as

$$
p[t]=\mathbb{I}\left(t=t_{a}\right) \bar{p}[t], \quad t=1, \ldots, T,
$$

where

$$
t_{a}=\underset{1 \leq t \leq T}{\arg \min } \mathbb{E}\left\{\left(a[t]-b\left[t_{b}\right]\right) \mathbb{I}(a[t] \leq \bar{p}[t])\right\},
$$

and for each $t=1, \ldots, T$, we have

$$
\begin{aligned}
\bar{p}[t]=\underset{p[t] \geq 0}{\arg \min } & \mathbb{E}\left\{\left(a[t]-b\left[t_{b}\right]\right) \mathbb{I}(a[t] \leq p[t])\right\} \\
& \times \mathbb{I}\left(\mathbb{E}\left\{\left(a[t]-b\left[t_{b}\right]\right) \mathbb{I}(a[t] \leq p[t])\right\} \leq 0\right) .
\end{aligned}
$$


The above Theorem is the direct result of the problem formulation in (19) and the definitions in (22) and (23). The values of $\bar{p}[1], \ldots, \bar{p}[T]$ depend on the statistical characteristics of the day-ahead and real-time market prices. Therefore, they can be calculated offline and used later at the time of bidding. Given the optimal price bid vector $\boldsymbol{p}$ from Theorem 5, the optimal energy bid vector $\boldsymbol{x}$ is readily obtained using Theorem 4 , where $t_{a, p}$ in (16) is replaced by $t_{a}$ in (22).

If the day-ahead market prices are in fact independent from the real-time market price at time $t_{b}$, then $\bar{p}[1]=\ldots=\bar{p}[T]=$ $\mathbb{E}\left\{b\left[t_{b}\right]\right\}$; and (21) reduces to (13), while (22) reduces to (12).

\section{Case Study}

Consider the PJM price data in Fig. 2; and assume that a time-shiftable load with one subload seeks to procure energy based on the bidding framework in Fig. 1. We assume that $\alpha_{1}=5: 00 \mathrm{PM}=17, \beta_{1}=10: 00 \mathrm{PM}=22$, and $e_{1}=5 \mathrm{MWh}$. We have $t_{b}=8: 00 \mathrm{PM}$ and $\mathbb{E}\left\{b\left[t_{b}\right]\right\}=\$ 41.07$. For Design I in Section III-B-1, the term $\mathbb{E}\left\{\left(a[t]-\mathbb{E}\left\{b\left[t_{b}\right]\right\}\right) \mathbb{I}(a[t] \leq\right.$ $\left.\left.\mathbb{E}\left\{b\left[t_{b}\right]\right\}\right)\right\}$ is obtained as $-\$ 7.21,-\$ 5.17,-\$ 4.60,-\$ 4.15$, $\$ 4.39$, and $-\$ 5.09$, for $t=17, \ldots, 22$. From (12), we have $t_{a}=$ 5:00 $\mathrm{PM}=17$. The bids to the day-ahead market are obtained as $p[17]=\$ 41.07$ and $x[17]=5 \mathrm{MWh}$; while $p[t]=x[t]=0$ for any $t \neq 17$. The expected energy procurement cost is calculated as $\$ 189.43$. For Design II in Section III-B-2, the bidding parameter $\bar{p}[t]$ in (23) is obtained as $\$ 44.01, \$ 44.62$, $\$ 42.50, \$ 32.14, \$ 29.68$, and $\$ 49.03$, for $t=17, \ldots, 22$. Accordingly, the terms $\mathbb{E}\left\{\left(a[t]-b\left[t_{b}\right]\right) \mathbb{I}(a[t] \leq \bar{p}[t])\right\}$ are obtained as $-\$ 3.49,-\$ 1.34,-\$ 0.41,-\$ 0.03,-\$ 0.02$, and $-\$ 1.75$. From (22), we have $t_{a}=5: 00 \mathrm{PM}=17$. The bids to the dayahead market are obtained as $p[17]=\$ 44.01$ and $x[17]=5$ MWh, with $p[t]=x[t]=0$ for any $t \neq 17$. The expected energy cost is calculated as $\$ 187.85$. We can see that relaxing the assumption on price independency between the day-ahead and real-time markets can further lower the cost.

As a baseline for comparison, if energy usage is evenly distributed from 5:00 PM to 10:00 PM and energy at each time slot is procured only from the day-ahead market at the clearing market price, then the expected cost of energy procurement is $\$ 211.15$. Compared to this baseline, Design I in Section III-B-1 reduces energy cost by $11.0 \%$. The energy cost further reduces by $0.8 \%$ when Design II in Section III-B-2 is used.

If energy is procured only from the day-ahead market at the hour with the lowest expected day-ahead market price, i.e., at $t=17$, then the expected energy procurement cost becomes $\$ 200.12$. Also if energy is procured only from the real-time market at the hour with the lowest expected real-time market price, i.e., at $t=20$, then the expected energy procurement cost becomes $\$ 205.33$. Designs I and II outperform both cases.

\section{The Case with Several Subloads}

In this section, we consider the general case where the time-shiftable load comprises of several smaller time-shiftable subloads, i.e., $L>1$. We consider two different scenarios to submit the bids. The first scenario is per-subload bidding, where for each subload $l$ and at each time slot $t$, we submit one bid pair $p_{l}[t]$ and $x_{l}[t]$ to the day-ahead market and one bid

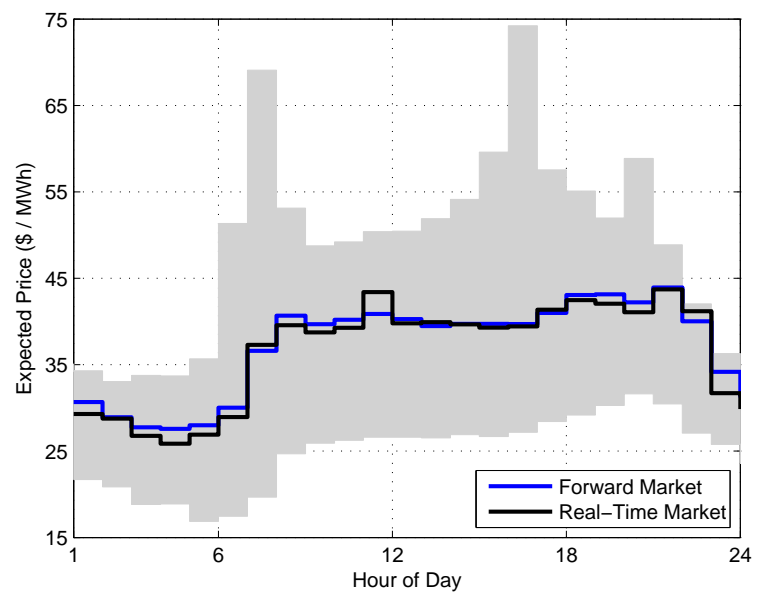

Fig. 2. Statistical characteristics of the day-ahead and real-time market prices at PJM for reported data from January 1 to June 30, 2013. The shaded area indicates the $90 \%$ confidence interval for day-ahead market prices. The data in this figure is used for the case studies in Sections III and IV.

$y_{l}[t]$ to the real-time market. The second scenario is single bidding, where, regardless of the number of subloads, we submit only one bid pair $p[t]$ and $x[t]$ to the day-ahead market and only one bid $y[t]$ to the real-time market. It is clear that by submitting per-subload bids we increase granularity and allow a more fine-grained bidding process. Therefore, the persubload bidding is always no worse than single bidding. As a result, there is no reason not to go for per-subload bidding, unless the time-shiftable load is mandated by the market to submit only a single bid despite having multiple subloads.

\section{A. When Per-Subload Bidding is Possible}

Under the per-subload bidding scenario, at each time slot $t$, the time-shiftable load submits price bids $p_{l}[t], \ldots, p_{L}[t]$ and energy bids $x_{l}[t], \ldots, x_{L}[t]$ to the day-ahead market and energy bids $y_{l}[t], \ldots, y_{L}[t]$ to the real-time market. Of course, for each subload $l$, there is no need for energy procurement outside the time frame $\left[\alpha_{l}, \beta_{l}\right]$; and we can trivially choose

$$
p_{l}[t]=x_{l}[t]=y_{l}[t]=0, \quad \begin{aligned}
t & =1, \ldots, \alpha_{l}-1, \\
t & =\beta_{l}+1, \ldots, T .
\end{aligned}
$$

Since separate bids are submitted for each subload, and with the assumption that the time-shiftable load is price taker, the problem of selecting the bids is essentially independent across different subloads. As a result, the optimal bids for each subload is obtained by following the exact same steps that we explained in Section III. For example, for the case of Design I, where the day-ahead and real-time market prices are independent, for each subload $l$, the optimal bids are

$$
\begin{array}{clrl}
p_{l}[t]=\mathbb{I}\left(t=t_{a, l}\right) \mathbb{E}\left\{b\left[t_{b, l}\right]\right\}, & t & =\alpha_{l}, \ldots, \beta_{l}, \\
x_{l}[t]=\mathbb{I}\left(t=t_{a, l}\right) e_{l}, & & t=\alpha_{l}, \ldots, \beta_{l}, \\
y_{l}[t]=\mathbb{I}\left(t=t_{b, l}\right)\left(e_{l}-X_{l}\right), & & t=\alpha_{l}, \ldots, \beta_{l},
\end{array}
$$

where

$$
t_{b, l}=\underset{\alpha_{l} \leq t \leq \beta_{l}}{\arg \min } \mathbb{E}\{b[t]\}
$$




$$
t_{a, l}=\underset{\alpha_{l} \leq t \leq \beta_{l}}{\arg \min } \mathbb{E}\left\{\left(a[t]-\mathbb{E}\left\{b\left[t_{b, l}\right]\right\}\right) \mathbb{I}\left(a[t] \leq \mathbb{E}\left\{b\left[t_{b, l}\right]\right\}\right)\right\},
$$

and

$$
X_{l}=\mathbb{I}\left(a[t] \leq \mathbb{E}\left\{b\left[t_{b, l}\right]\right\}\right) e_{l} .
$$

The optimal bids for the case of Design II with no assumption on market price independency can be obtained similarly by extending the related results in Theorems 1, 4, and 5 .

Let us define $\mathcal{L} \triangleq\{1, \ldots, L\}$. From (25)-(30), for each time slot $t$, the time-shiftable load submits exactly $L_{a}[t]$ bids to the day-ahead market and $L_{b}[t]$ bids to the real-time market, where $L_{a}[t]$ and $L_{b}[t]$ are the cardinalities of sets

$$
\begin{aligned}
\mathcal{L}_{a}[t] & =\left\{l \mid l \in \mathcal{L}, t=t_{a, l}\right\}, \\
\mathcal{L}_{b}[t] & =\left\{l \mid l \in \mathcal{L}, t=t_{b, l}\right\} .
\end{aligned}
$$

Once the day-ahead market is settled and the market clearing price $a[t]$ is realized, the total amount of power that is procured from the day-ahead market at time slot $t$ is calculated as

$$
X[t]=\sum_{l \in \mathcal{L}_{a}} \mathbb{I}\left(a[t] \leq \mathbb{E}\left\{b\left[t_{b, l}\right]\right\}\right) e_{l} .
$$

It is interesting to note that $X[t]$ in (33) is in fact the demand function of the time-shiftable load at time slot $t$, a measure that is widely used in electricity market analysis [4].

As a case study, consider the example in Fig. 3, where we have visualized the subload parameters of a time-shiftable load with $L=10$ subloads. Each line segment represents one subload $l$, where the start and the end points denote parameters $\alpha_{1}$ and $\beta_{l}$, respectively. For example, for $l=1$, we have $\alpha_{1}=17$ and $\beta_{1}=22$; and for $l=2$, we have $\alpha_{2}=11$ and $\beta_{2}=19$. The optimal demand bids to the day-ahead market for the example time-shiftable load in Fig. 3, based on both Design I and Design II, are shown in Fig. 4. Here, again, we assume that the electricity market prices are as in Fig. 2.

First, consider the demand bids in the first row in Fig. 4, which are obtained based on Design I. There are non-zero demand bids at six different hours: $\mathcal{L}_{a}[7]=\{4,5,9\}, \mathcal{L}_{a}[8]=$ $\{8\}, \mathcal{L}_{a}[14]=\{3\}, \mathcal{L}_{a}[16]=\{2,6,10\}, \mathcal{L}_{a}[17]=\{1\}$, and $\mathcal{L}_{a}[22]=\{7\}$. As an example, consider the demand bid at hour $t=7: 00$ PM. It consists of three segments, including the segment for subload number 4 . This is because $t_{a, 4}=7$. To see why, note that $\alpha_{4}=5$ and $\beta_{4}=12$; and the term $\mathbb{E}\left\{\left(a[t]-\mathbb{E}\left\{b\left[t_{b, 4}\right]\right\}\right) \mathbb{I}\left(a[t] \leq \mathbb{E}\left\{b\left[t_{b, 4}\right]\right\}\right)\right\}$ is obtained as $\$ 2.80,-\$ 2.82,-\$ 3.64,-\$ 2.36,-\$ 0.92,-\$ 1.48,-\$ 1.21$, and $\$ 1.16$, for $t=5, \ldots, 12$. Also, from (28), we have $t_{b, 4}=5$, $t_{b, 5}=6, t_{b, 9}=4$; and $\mathbb{E}\left\{b\left[t_{b, 4}\right]\right\}=\$ 26.91, \mathbb{E}\left\{b\left[t_{b, 5}\right]\right\}=$ $\$ 28.95$, and $\mathbb{E}\left\{b\left[t_{b, 9}\right]\right\}=\$ 25.85$. For the demand bid at hour $t=7$, the price bids are at $\$ 25.85, \$ 26.91, \$ 28.95$; and the energy bids are at $e_{5}=2, e_{5}+e_{4}=8 \mathrm{MWh}$, and $e_{5}+e_{4}+e_{9}=$ $12 \mathrm{MWh}$. As another example, consider the demand bid at hour $t=16=4: 00$ PM, which has only one segment. Since $t_{b, 2}=t_{b, 6}=t_{b, 10}=15$, the price bid is at $\mathbb{E}\{b[15]\}=\$ 39.30$ and the energy bid is at $e_{2}+e_{6}+e_{10}=15 \mathrm{MWh}$.

Next, consider the demand bids in the second row in Fig. 4, which are obtained based on Design II. This time, there are non-zero demand bids at seven different hours: $\mathcal{L}_{a}[3]=$ $\{9\}, \mathcal{L}_{a}[5]=\{4\}, \mathcal{L}_{a}[14]=\{3\}, \mathcal{L}_{a}[15]=\{8\}, \mathcal{L}_{a}[16]=$

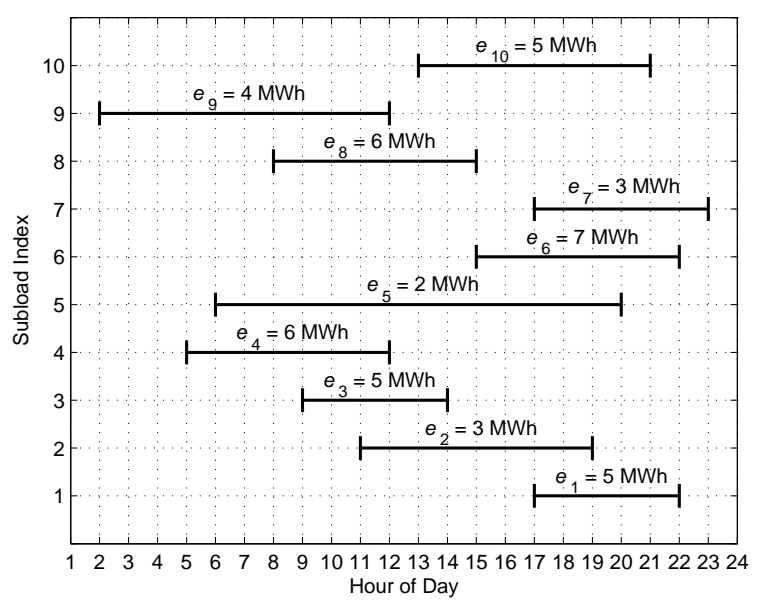

Fig. 3. Parameters of an example time-shiftable load with $L=10$ subloads. For each subload $l$, the line segment starts from $\alpha_{l}$ and ends at $\beta_{l}$.

$\{2,6,10\}, \mathcal{L}_{a}[17]=\{1\}$, and $\mathcal{L}_{a}[18]=\{5,7\}$. We can see that there are some similarities but also some major differences between the demand bids that are obtained based on Design I and Design II. For example, the bid segment corresponding to subload number 4 has now moved from 7:00 PM to time slot $t=t_{a, 4}=5: 00$ PM. From Theorem 5, the price component for the demand bid for this subload is $\bar{p}_{4}\left[t_{a, 4}\right]=\$ 18.90$.

When obtaining the optimal bids based on Designs I and II in Sections III-B-1 and III-B-2, the expected cost of energy procurement is calculated as $\$ 1,632$ and $\$ 1,595$, respectively. For the baseline scenario that we defined in Section III-C, the expected cost of energy procurement is $\$ 1,835$. Compared to the baseline, Design I reduces energy cost by $11.1 \%$. The energy cost further reduces by $2.3 \%$ when Design II is used.

If energy is procured only from the day-ahead market at the hours with the lowest expected day-ahead market price, then the expected energy procurement cost becomes $\$ 1,669$. Also if energy is procured only from the real-time market at the hours with the lowest expected real-time market price, then the expected energy procurement cost becomes $\$ 1,639$. Therefore, Designs I and II outperform both cases in this example.

\section{B. When Per-Subload Bidding is not Possible}

Based on the per-subload bidding mechanism in Section IV-A, each subload can potentially create a new segment in the total demand bid. As a result, the demand bids at certain hours may have multiple segments, as in the cases of hour 7:00 $\mathrm{AM}$ and hour 6:00 PM in Fig. 4, with three and two segments, respectively. This is an interesting observation, because in practice, there is typically a limit on the number of segments (stairs) in the demand or supply bids. For example, in the California ISO day-ahead market, the number of bid segments is limited to 10. Therefore, it is natural to ask the following question: How binding, in terms of demand bidding efficiency, is the limit on the number of segments in each bid? Clearly, having the limit at 10 is no worse than having it at 9, and having the limit at 9 is no worse than having it at 8 , and so on and so forth. Therefore, if any such limit is going to be binding, then we should see it in the most restrictive scenario 

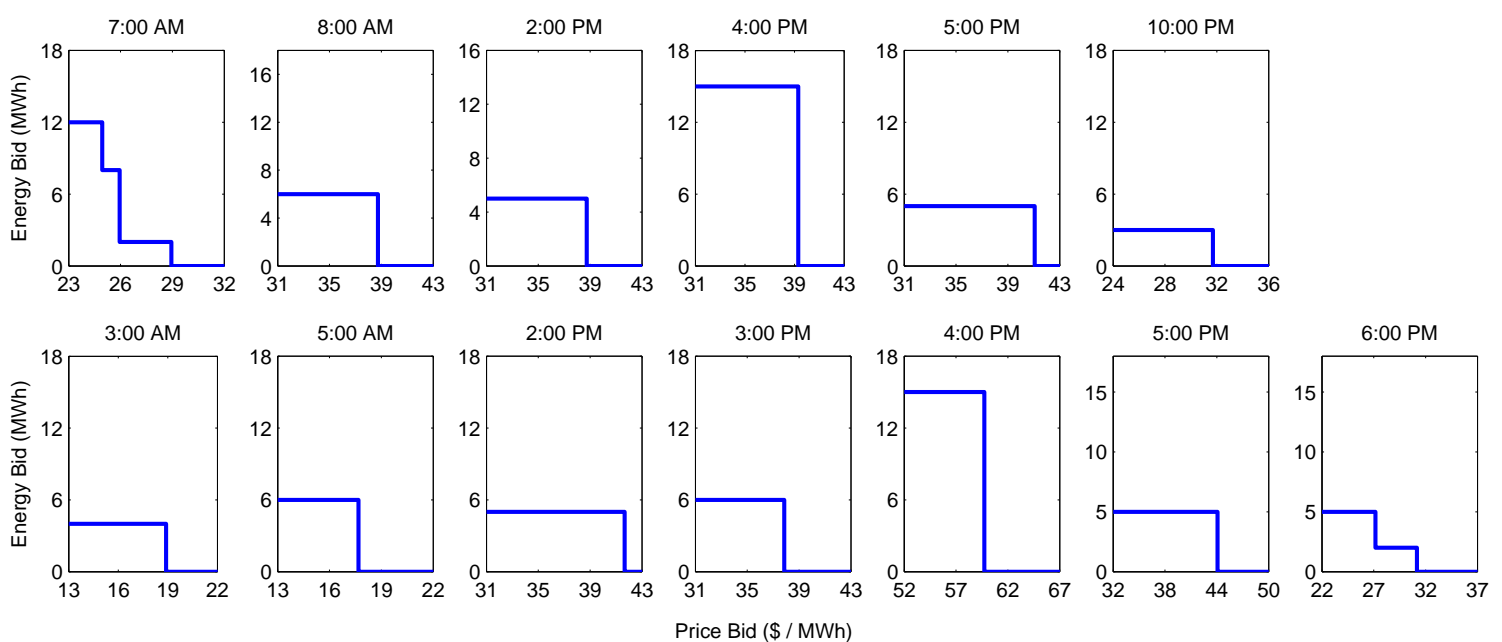

Fig. 4. The optimal demand bids to the day-ahead market for the example time-shiftable load in Fig. 3 with $L=10$ subloads. The first row shows the results based on Design I. The second row shows the results based on Design II. Only the hours with non-zero demand bids are shown here.

where the demand bid is limited to only one segment. Hence, to gain insights, we examine such highly restrictive scenario in this section. That is, we consider the case where the market mandates each time-shiftable load to submit only one bid for each time slot, regardless of the number of its subloads.

Because of the linear property of Fixed Demand bids and the price-taker assumption, submitting $L$ different bids $y_{1}[t]$, $\ldots, y_{L}[t]$ to real-time market at time slot $t$ results in an identical energy procurement as if we submit a single bid

$$
y[t]=\sum_{l=1}^{L} y_{l}[t]
$$

Thus, by conducting a backward induction analysis similar to the one in the Proof of Theorem 1, we can show that the single optimal bid to the real-time market is obtained as

$$
y[t]=\sum_{l=1}^{L} y_{l}[t]=\sum_{l=1}^{L} \mathbb{I}\left(t=t_{b, l}\right)\left(e_{l}-X_{l}\right),
$$

where $X_{l}$ is the portion of the total energy that is procured from the day-ahead market for consumption at subload $l$ at time slot $t$. For each subload $l$, notation $t_{b, l}$ is as in (28).

Next, we note that once the day-ahead market is cleared, the time-shiftable load operator must distribute the total procured energy $\mathbb{I}(a[t] \leq p[t]) x[t]$ across its subloads. This can be done by using auxiliary decision variables $x_{1}[t], \ldots, x_{L}[t]$, where

$$
x[t]=\sum_{l=1}^{L} x_{l}[t]
$$

and for each subload $l=1, \ldots, L$, we must have

$$
X_{l}=\sum_{t=\alpha_{l}}^{\beta_{l}} \mathbb{I}(a[t] \leq p[t]) x_{l}[t] \leq e_{l}, \quad \forall a[1], \ldots, a[T]
$$

Therefore, the stochastic optimization problem for selecting the bids to the day-ahead market can be formulated as

$$
\begin{aligned}
\underset{\boldsymbol{p}, \boldsymbol{x}}{\operatorname{Min}} & \mathbb{E}\left\{\sum_{t=1}^{T} \mathbb{I}(a[t] \leq p[t]) a[t] x[t]\right. \\
& \left.+\sum_{l=1}^{L} b\left[t_{b, l}\right]\left(e_{l}-\sum_{t=1}^{T} \mathbb{I}(a[t] \leq p[t]) x_{l}[t]\right)\right\}
\end{aligned}
$$

S.t. $\quad \sum_{t=\alpha_{1}}^{\beta_{1}} \mathbb{I}(a[t] \leq p[t]) x_{1}[t] \leq e_{1}, \forall a[1], \ldots, a[T]$,

$$
\begin{aligned}
& \sum_{t=\alpha_{L}}^{\beta_{L}} \mathbb{I}(a[t] \leq p[t]) x_{L}[t] \leq e_{L}, \forall a[1], \ldots, a[T], \\
& x_{l}[t]=0, \quad l=1, \ldots, L, t \notin\left[\alpha_{l}, \beta_{l}\right], \\
& x[t]=\sum_{l=1}^{L} x_{l}[t], \quad t=1, \ldots, T .
\end{aligned}
$$

where for each time slot $t$, we define $\boldsymbol{x}_{l}[t] \triangleq x_{l}[1], \ldots, x_{l}[T]$. As in problem (5), we can replace the infinite number of inequalities for each subload with a single inequality constraint. We can also eliminate the equality constraints on the last line by substituting any $x[t]$ with $\sum_{l=1}^{L} x_{l}[t]$ for all $t=1, \ldots, T$. As for the objective function in (38), after reordering the terms, and once we remove all fixed terms, it becomes

$$
\sum_{l=1}^{L} \sum_{t=1}^{T} \mathbb{E}\left\{\left(a[t]-b\left[t_{b, l}\right]\right) \mathbb{I}(a[t] \leq p[t])\right\} x_{l}[t] .
$$

Therefore, we can rewrite problem (38) as

$$
\begin{array}{cl}
\underset{\boldsymbol{p}, \boldsymbol{x}_{1}, \ldots, \boldsymbol{x}_{L}}{\operatorname{Min}} & \sum_{l=1}^{L} \sum_{t=1}^{T} \mathbb{E}\left\{\left(a[t]-b\left[t_{b, l}\right]\right) \mathbb{I}(a[t] \leq p[t])\right\} x_{l}[t] \\
\text { S.t. } \quad & x_{l}[t]=0, \quad l=1, \ldots, L, t \notin\left[\alpha_{l}, \beta_{l}\right], \\
& \sum_{t=\alpha_{l}}^{\beta_{l}} x_{l}[t] \leq e_{l}, \quad l=1, \ldots, L .
\end{array}
$$


Problem (40) generalizes problem (5). If we assume that the prices in the day-ahead and real-time markets are independent, then we can accordingly generalize Theorem 2 . However, such generalization provides only some bounds for $p[t]$ rather than an exact closed-form solution as in Theorem 2. Therefore, next, we directly move to the general case of Design II, where there is no particular assumption on the statistical characteristics of the day-ahead and real-time market prices.

Suppose the price bid vector $\boldsymbol{p}$ is already selected. This removes the coupling across different subloads in problem (40) and allows us to decompose problem (40) into $L$ separate subproblems. Given $\boldsymbol{p}$, for each $l=1, \ldots, L$, we must solve

$$
\begin{aligned}
\underset{\boldsymbol{x}_{l}}{\operatorname{Min}} & \sum_{t=\alpha_{l}}^{\beta_{l}} \mathbb{E}\left\{\left(a[t]-b\left[t_{b, l}\right]\right) \mathbb{I}(a[t] \leq p[t])\right\} x_{l}[t] \\
\text { S.t. } & \sum_{t=\alpha_{l}}^{\beta_{l}} x_{l}[t] \leq e_{l} .
\end{aligned}
$$

Next, let us denote the coefficient of variable $x_{l}[t]$ in (41) as

$$
\mathbb{C}_{l}(t, p[t])=\mathbb{E}\left\{\left(a[t]-b\left[t_{b, l}\right]\right) \mathbb{I}(a[t] \leq p[t])\right\} .
$$

Similar to the case in Theorem 4, we can show that for a given $\boldsymbol{p}$ and for each time slot $t$, at optimality, we have

$$
x_{l}[t]=\mathbb{I}\left(t=t_{a, l, \boldsymbol{p}}\right) \mathbb{I}\left(\mathbb{C}_{l}\left(t_{a, l, \boldsymbol{p}}, p\left[t_{a, l, \boldsymbol{p}}\right]\right) \leq 0\right) e_{l},
$$

where

$$
t_{a, l, \boldsymbol{p}}=\underset{\alpha_{l} \leq t \leq \beta_{l}}{\arg \min } \mathbb{C}_{l}(t, p[t]) .
$$

The optimal objective value in (41) for the given $\boldsymbol{p}$ becomes

$$
\mathbb{I}\left(\mathbb{C}_{l}\left(t_{a, l, \boldsymbol{p}}, p\left[t_{a, l, \boldsymbol{p}}\right]\right) \leq 0\right) \mathbb{C}_{l}\left(t_{a, l, \boldsymbol{p}}, p\left[t_{a, l, \boldsymbol{p}}\right]\right) e_{l} .
$$

Therefore, we can rewrite problem (41) over $\boldsymbol{p}$ as

$$
\begin{gathered}
\underset{\boldsymbol{p}}{\operatorname{Min}} \sum_{l=1}^{L} \mathbb{I}\left(\mathbb{C}_{l}\left(t_{a, l, \boldsymbol{p}}, p\left[t_{a, l, \boldsymbol{p}}\right]\right) \leq 0\right) \mathbb{C}\left(t_{a, l, \boldsymbol{p}}, p\left[t_{a, l \boldsymbol{p}}\right]\right) e_{l} \\
=\underset{\boldsymbol{p}}{\operatorname{Min}} \sum_{l=1}^{L} \min _{\alpha_{l} \leq t \leq \beta_{l}} \mathbb{C}_{l}(t, p[t]) \mathbb{I}\left(\mathbb{C}_{l}(t, p[t]) \leq 0\right) e_{l} \\
=\underset{\boldsymbol{p}}{\operatorname{Min}} \sum_{l=1}^{L} \min _{1 \leq t \leq T} \mathbb{C}_{l}(t, p[t]) \mathbb{I}\left(\mathbb{C}_{l}(t, p[t]) \leq 0\right) \\
\times \mathbb{I}\left(\alpha_{l} \leq t \leq \beta_{l}\right) e_{l} .
\end{gathered}
$$

Because of the summation over $l$, we cannot switch the two min operators on the last line in (46). Therefore, unlike problem (19), problem (46) is not separable. Nevertheless, we can still follow the approach in Section III-B-2 and decompose problem (46) in order to obtain a sub-optimal solution:

$$
\begin{array}{rl}
p[t]=\underset{p[t] \geq 0}{\arg \min } \sum_{l=1}^{L} & \mathbb{E}\left\{\left(a[t]-b\left[t_{b, l}\right]\right) \mathbb{I}(a[t] \leq p[t])\right\} \\
& \mathbb{I}\left(\mathbb{E}\left\{\left(a[t]-b\left[t_{b, l}\right]\right) \mathbb{I}(a[t] \leq p[t])\right\} \leq 0\right),
\end{array}
$$

where $t=1, \ldots, T$. Given the price bids in (47), the corresponding optimal energy bids $\boldsymbol{x}$ are readily obtained using the results in (43) and (44) and the relationship in (36).
In practice, the optimality gap for the above sub-optimal solutions is very small. This can be verified by comparing the expected energy procurement cost when we use (43) and (47) with the expected energy procurement cost when we use the optimal per-subload bidding solutions in Section IV-A. For example, recall from Section IV-A that under per-subload bidding, the total expected energy procurement cost for the time-shiftable load in Fig. 3 is $\$ 1,595$. Since per-subload bidding is always no worse than single bidding, this number gives a lower bound for the objective value of the minimization problem in (40). Now, if we use the bids in (43) and (47), the total expected energy procurement power again becomes $\$ 1,595$. Therefore, in this example, the optimality gap is zero.

\section{Additional Case Studies}

\section{A. Other Price Data}

Again, consider the subloads in Fig. 3. For the results in Sections IV-A and IV-B, we used the price data in Fig. 2. Next, we re-examine the results for ten different 6-months PJM price data sets, as in Fig. 5. For each year, two sets of results are shown here, one based on the data from January 1 to June 30 and another one based on the data from July 1 to December 31. The Baseline is defined as in Section III-C.

We can see that all designs highly outperform the baseline, while Design II slightly outperforms Design I in all cases. Interestingly, the difference between per-subload bidding and single bidding is relatively minor, suggesting that a mandate on submitting only a single bid does not significantly increase the time-shiftable load's energy procurement cost. On average, and across all ten price cases, the cost of energy procurement for the Baseline, Single Bid - Design I, Per-Subload Bid Design I, Single Bid - Design II, and Per-Subload Bid Design II, is $\$ 1,966, \$ 1,669, \$ 1,662, \$ 1,623$, and $\$ 1,617$, respectively.

If energy is procured only from the day-ahead market at the hours with the lowest expected day-ahead market price, then on average, i.e., across all ten price cases, the expected energy cost is $\$ 1,683$. If energy is procured only from the real-time market at the hours with the lowest expected real-time market price, then on average, the expected energy cost is $\$ 1,672$. We can see that Designs I and II outperform both cases.

\section{B. Other Subload Configurations}

Next, we examine 20 scenarios with different subload configurations. For each scenario, we randomly choose parameters $\alpha_{l}, \beta_{l}$, and $e_{l}$ for $L=10$ subloads. Furthermore, for each scenario, we assume that the price data is randomly selected from the ten different 6-months PJM price data sets that we discussed in Section V-A. The results are shown in Fig. 6. We can see that the trends are similar to those that we previously saw for the particular subload configuration of Fig. 3. This suggests that the advantages of the proposed design is not specific to a particular subload configuration. On average, and across all 20 randomly generated subload configuration scenarios, the cost of energy procurement for the Baseline, Single Bid - Design I, Per-Subload Bid - Design I, Single Bid - Design II, and Per-Subload Bid Design II, is $\$ 2,339$, \$1,966, $\$ 1,956, \$ 1,910$, and $\$ 1,904$, respectively. 


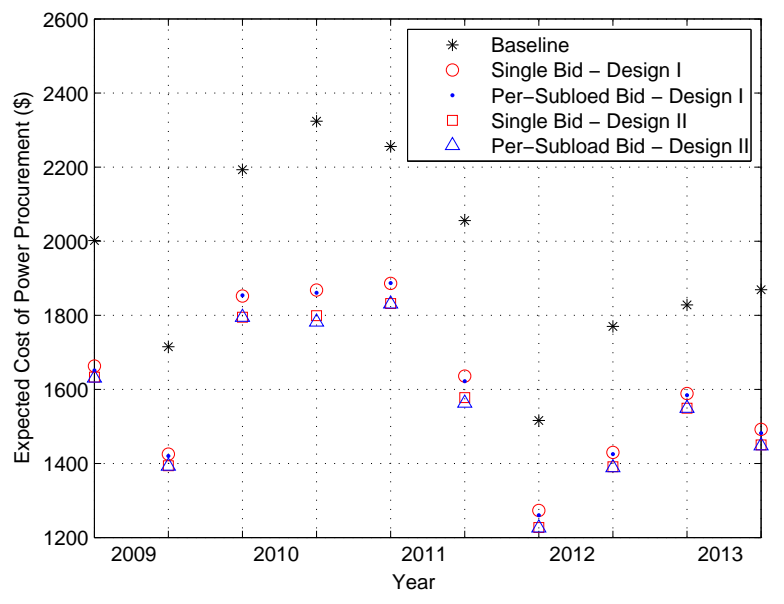

Fig. 5. The expected energy procurement cost of the time-shiftable load with subloads as in Fig. 3 based on ten different 6-months PJM price data sets.

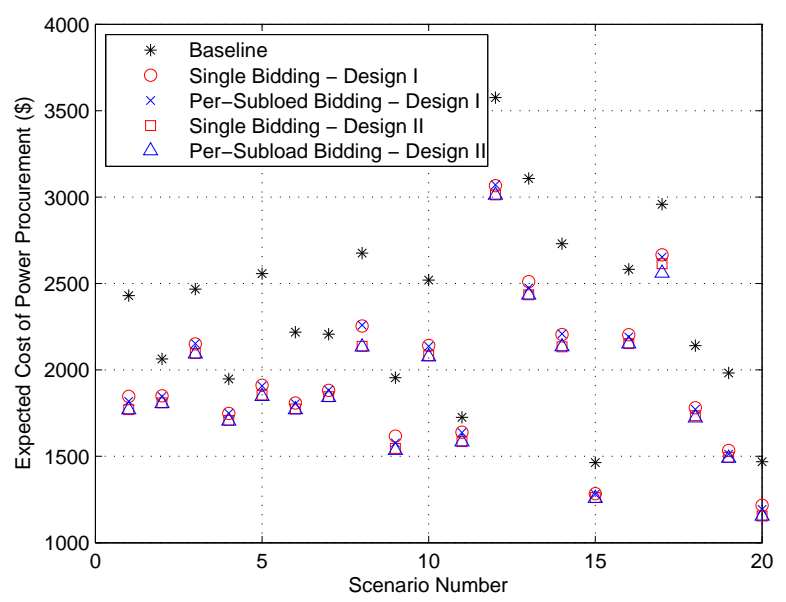

Fig. 6. The expected energy procurement cost of a time-shiftable load with 20 different randomly generated subload configurations, where $L=10$.

If energy is procured only from the day-ahead market at the hours with the lowest expected day-ahead market price, then, on average, i.e., across all 20 randomly generated subload configuration scenarios, the expected energy cost is $\$ 1,997$. If energy is procured only from the real-time market at the hours with the lowest expected real-time market price, then, on average, the expected energy cost is $\$ 1,958$. Single Bid Design I and particularly the two approaches for Design II outperform both cases. We see that restricting the number of bids to only one segment may slightly hurt the bidder in some load configurations. Although, even in those cases, Design II can still provide a very efficient demand bidding performance.

\section{Increasing the Number of Subloads}

The results when we increase the number of subloads are shown in Fig. 7, where each point denotes the average expected energy procurement cost across 20 different randomly generated subload configuration scenarios. For example, the results for $L=10$ are the average of the results in Fig. 6 . As we increase $L$, the relative performance of the various designs is more or less maintained. With $L=100$ subloads,

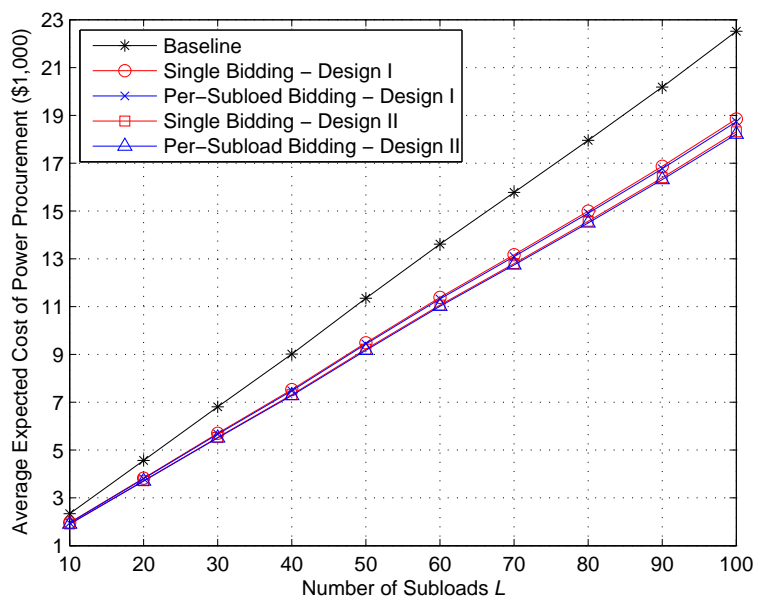

Fig. 7. The impact of increasing the number of subloads. Each point indicates the average expected energy procurement cost across 20 random scenarios.

the average expected energy procurement cost for the Baseline, Single Bid - Design I, Per-Subload Bid - Design I, Single Bid Design II, and Per-Subload Bid Design II, is $\$ 22,515, \$ 18,847$, $\$ 18,730, \$ 18,309, \$ 18,213$, respectively.

If $L=100$ and energy is procured only from the day-ahead market at the hours with the lowest expected day-ahead market price, then, on average, i.e., across all 20 randomly generated subload configuration scenarios, the expected energy cost is $\$ 19,052$. If energy is procured only from the real-time market at the hours with the lowest expected real-time market price, then on average, the expected energy cost is $\$ 18,721$. The two approaches for Design II always outperform both cases.

\section{Vi. CONClusions And Future Work}

We developed a multi-stage stochastic optimization framework, together with several closed-form solutions, to optimally select the demand bids for time-shiftable loads. Both persubload bidding and single bidding were considered. Using PJM price data, the proposed designs were tested in various case studies. The impact of different price scenarios, subload configurations, and bidding rules were investigated. We observed that, while per-subload bidding outperforms single bidding, the performance loss for the latter is typically small. Also, in general, it is preferred to take into account the statistical dependence between the day-ahead and real-time market prices. The designs in this paper can help large consumers, LSEs, and utilities to better exploit their time-shiftable load potentials to lower their energy costs in electricity markets.

The analysis and results in this paper can be extended in several directions. First, the focus throughout this work was limited to the most generic form of time-shiftable loads. However, the same methodology can be applied to incorporate more details about loads and their characteristics. For example, one can take into consideration the limits on power usage at each time slot, ramping constraints, and whether the timeshiftable load is interruptible or uninterruptible. Second, it is interesting to see how the results may change when we relax the price-taker assumption. Such extension is particularly important if we increase the penetration of time-shiftable loads 
in the electricity market to a level so that they become pricemaker, i.e., the time-shift in their demand creates noticeable change in the price values. Third, when it comes to pricemaker loads, such as large utilities whose service territory covers several trading nodes within a transmission network, the congestion effect in the underlying transmission network topology has to be considered in the bidding strategy.

\section{REFERENCES}

[1] ERCOT, http://www.ercot.com/content/services/programs/load/Load_ Participation_in_the_ERCOT_Nodal_Market_v1_0_061107.pdf.

[2] PJM, http://www.pjm.com/ /media/committees-groups/task-forces/ttf/ 20120523/20120523-day-ahead-market-modeling-presentation.ashx.

[3] California ISO, http://www.caiso.com/1c78/1c788230719c0.pdf.

[4] M. Shahidehpour, H. Yamin, and Z. Li, Market Operations in Electric Power Systems. New York, NY: John Wiley \& Sons, 2002.

[5] X. Geng and P. P. Khargonekar, "Electric vehicles as flexible loads: Algorithms to optimize aggregate behavior," in Proc of IEEE Conference on Smart Grid Communications, Tainan City, Taiwan, nov. 2012.

[6] S. Shao, T. Zhang, M. Pipattanasomporn, and S. Rahman, "Impact of TOU rates on distribution load shapes in a smart grid with PHEV penetration," in Proc. of IEEE PES Transmission and Distribution Conference and Exposition, New Orleans, LA, 2010 Apr.

[7] S. Meyn, P. Barooah, A. Busic, and J. Ehren, "Ancillary service to the grid from deferrable loads: The case for intelligent pool pumps in Florida," in Proc. of IEEE Conference on Decision and Control, Florence, Italy, 2013 Dec.

[8] G. Marks, E. Wilcox, D. Olsen, and S. Goli, "Opportunities for demand response in California agricultural irrigation: A scoping study," Technical Report, Lawrence Berkeley National Laboratory, Jan. 2013.

[9] M. Ghamkhari and H. Mohsenian-Rad, "Energy and performance management of green data centers: A profit maximization approach," IEEE Trans. on Smart Grid, vol. 4, no. 2, pp. 1017-1025, Jun. 2013.

[10] B. Aksanli, J. Venkatesh, L. Zhang, and T. Rosing, "Utilizing green energy prediction to schedule mixed batch and service jobs in data centers," in Proc. of ACM Workshop on Power-Aware Computing and Systems, Cascais, Portugal, Oct. 2011.

[11] Z. Liu, I. Liu, S. Low, and A. Wierman, "Pricing data center demand response," in Proc. of ACM Sigmetrics, Austin, TX, 2014 Jun.

[12] A. Gholian, H. Mohsenian-Rad, Y. Hua, and J. Qin, "Optimal industrial load control in smart grid: A case study for oil refineries," in Proc. of IEEE PES General Meeting, Vancouver, Canada, Jul. 2013.

[13] P. Yang, G. Tang, and A. Nehorai, "A game-theoretic approach for optimal time-of-use electricity pricing," IEEE Trans. on Power Systems, vol. 28, no. 2, pp. 884-892, May 2013.

[14] Z. Yu, L. Jia, M. C. Murphy-Hoye, A. Pratt, and L. Tong, "Modeling and stochastic control for home energy management," IEEE Trans. on Smart Grid, vol. 4, no. 4, pp. 2244-2255, Dec. 2013.

[15] A. S. Al-Sumaiti, M. H. Ahmed, and M. M. A. Salama, "Electric power components and systems," Smart Home Activities: A Literature Review, vol. 42, no. 3-4, pp. 294-305, Feb. 2014.

[16] H. Mohsenian-Rad and A. Leon-Garcia, "Optimal residential load control with price prediction in real-time electricity pricing environments," IEEE Trans. on Smart Grid, vol. 1, no. 2, pp. 120-133, Sep. 2010.

[17] H. Mohsenian-Rad, V. Wong, J. Jatskevich, R. Schober, and A. LeonGarcia, "Autonomous demand side management based on gametheoretic energy consumption scheduling for the future smart grid," IEEE Trans. on Smart Grid, vol. 1, no. 3, pp. 320-331, Dec. 2010.

[18] Z. Zhu, J. Tang, S. Lambotharan, W. Chin, and Z. Fan, "An integer linear programming based optimization for home demand-side management in smart grid," in Proc. of IEEE PES ISGT, Washington, DC, Jan. 2012.

[19] X. Chen, T. Wei, and S. Hu, "Uncertainty-aware household appliance scheduling considering dynamic electricity pricing in smart home," IEEE Trans. on Smart Grid, vol. 4, no. 2, pp. 932-941, Jun. 2013.

[20] Z. Chen, L. Wu, and Y. Fu, "Real-time price-based demand response management for residential appliances via stochastic optimization," IEEE Trans. on Smart Grid, vol. 3, no. 4, pp. 1822-1831, Dec. 2012.

[21] S. Han, S. Han, and K. Sezaki, "Development of an optimal vehicle-togrid aggregator for frequency regulation," IEEE Trans. on Smart Grid, vol. 1, no. 1, pp. 65-72, Jun. 2010.

[22] A. Papavasiliou and S. S. Oren, "Supplying renewable energy to deferrable loads: Algorithms and economic analysis," in Proc. of IEEE PES General Meeting, Minneapolis, MN, Jul. 2010.
[23] G. Ghanavati and S. Esmaeili, "Optimal bidding strategies for load server entities in electric power markets," in Proc. of IEEE Int. Conference on Computer and Electrical Engineering, Phuket, Thailand, Dec. 2008.

[24] M. Ghazvini, P. Faria, H. Morais, Z. Vale, and S. Ramos, "Stochastic framework for strategic decision-making of load-serving entities for dayahead market," in Proc. of IEEE PowerTech, Grenoble, France, 2013.

[25] A. Kian, J. B. Cruz, and R. J. Thomas, "Bidding strategies in oligopolistic dynamic electricity double-sided auctions," IEEE Trans. on Power Systems, vol. 20, no. 1, pp. 50-58, Feb. 2005.

[26] A. B. Philpott and E. Pettersen, "Optimizing demand-side bids in dayahead electricity markets," IEEE Trans. on Power Systems, vol. 21, no. 2, pp. 488-498, May 2006.

[27] L. Jiang and S. Low, "Multi-period optimal energy procurement and demand response in smart grid with uncertain supply," in Proc. of IEEE Conference on Decision and Control, Orlando, FL, Dec. 2011.

[28] L. Huang, J. Walrand, and K. Ramchandran, "Optimal power procurement and demand response with quality-of-usage guarantees," in Proc. of IEEE PES General Meeting, San Diego, CA, Jul. 2012.

[29] M. C. Caramanis and J. M. Foster, "Uniform and complex bids for demand response and wind generation scheduling in multi-period linked transmission and distribution markets," in Proc. of IEEE Conference on Decision and Control, Orlando, FL, Dec. 2011.

[30] K. Marti, Stochastic Optimization Methods. Springer, 2005.

\section{APPENDIX}

\section{A. Proof of Theorem 1}

Using backward induction from dynamic programming, we start from the last bidding stage at time slot $T$ and go backward in time to see how the bids are selected. Recall that, when it comes to the time to submit the last bid, we already know the values of $X$ and $y[1], \ldots, y[T-1]$, where

$$
0 \leq X+\sum_{t=1}^{T-1} y[t] \leq e_{1} .
$$

Therefore, in order to assure procuring total energy $e_{1}$ before deadline $T$, the only acceptable bidding scenario is to choose

$$
y[T]=e_{1}-X-\sum_{t=1}^{T-1} y[t] .
$$

Next, we move backward in time and examine the choice of bid $y[T-1]$. At this stage, given $X$ and $y[1], \ldots, y[T-2]$, and also based on the knowledge that the bid in the last stage will be chosen as in (49), we need to select $y[T-1]$ to minimize the expected value of the cost of procuring power. Therefore, we need to solve the following optimization problem

$$
\begin{array}{ll}
\operatorname{Min}_{y[T-1]} & \left(\sum_{t=1}^{T} \mathbb{I}(a[t] \leq p[t]) a[t] x[t]\right)+\left(\sum_{t=1}^{T-1} b[t] y[t]\right) \\
& +\mathbb{E}\{b[T-1] y[T-1] \\
& \left.+b[T]\left(e_{1}-X-\sum_{t=1}^{T-2} y[t]-y[T-1]\right)\right\} \\
\text { S.t. } \quad 0 \leq y[T-1] \leq e_{1}-X-\sum_{t=1}^{T-2} y[t] .
\end{array}
$$

After reordering the terms in the objective function and once we remove any term that does not depend on the optimization 
variable $y[T-1]$, problem $(50)$ reduces to

$$
\begin{array}{cl}
\underset{y[T-1]}{\operatorname{Min}} & (\mathbb{E}\{b[T-1]\}-\mathbb{E}\{b[T]\}) y[T-1] \\
\text { S.t. } & 0 \leq y[T-1] \leq e_{1}-X-\sum_{t=1}^{T-2} y[t] .
\end{array}
$$

If $\mathbb{E}\{b[T-1]\} \leq \mathbb{E}\{b[T]\}$, then, at optimality, we have

$$
y[T-1]=e_{1}-X-\sum_{t=1}^{T-2} y[t] \quad \Rightarrow \quad y[T]=0 .
$$

Otherwise, we have

$$
y[T-1]=0 \quad \Rightarrow \quad y[T]=e_{1}-X-\sum_{t=1}^{T-2} y[t] .
$$

Therefore, any shortage of energy $e_{1}-X-\sum_{t=1}^{T-2} y[t]$ that is realized before the last two time slots will be purchased in full at the time slot with the lowest expected market price.

Next, we examine the optimal choice of bid $y[T-2]$. At this stage, we need to solve the following optimization problem:

$$
\begin{aligned}
& \underset{y[T-2]}{\operatorname{Min}} \mathbb{E}\{b[T-2] y[T-2] \\
&+ b[T-1] \mathbb{I}(\mathbb{E}\{b[T-1]\} \leq \mathbb{E}\{b[T]\}) \\
& \times\left(e_{1}-X-\sum_{t=1}^{T-3} y[t]-y[T-2]\right) \\
&+ b[T](1-\mathbb{I}(\mathbb{E}\{b[T-1]\} \leq \mathbb{E}\{b[T]\})) \\
&\left.\times\left(e_{1}-X-\sum_{t=1}^{T-3} y[t]-y[T-2]\right)\right\}
\end{aligned}
$$

S.t $\quad 0 \leq y[T-2] \leq e-X-\sum_{t=1}^{T-3} y[t]$.

After removing the fixed terms, the objective function becomes

$$
\begin{aligned}
& (\mathbb{E}\{b[T-2]\}-\mathbb{E}\{b[T-1]\} \mathbb{I}(\mathbb{E}\{b[T-1]\} \leq \mathbb{E}\{b[T]\}) \\
& -\mathbb{E}\{b[T]\}(1-\mathbb{I}(\mathbb{E}\{b[T-1]\} \leq \mathbb{E}\{b[T]\}))) y[T-2] \\
& =(\mathbb{E}\{b[T-2]\}-\mathbb{E}\{b[T]\}-(\mathbb{E}\{b[T-1]\} \leq \mathbb{E}\{b[T]\}) \\
& \quad \times \mathbb{I}(\mathbb{E}\{b[T-1]\} \leq \mathbb{E}\{b[T]\})) y[T-2] \\
& =(\mathbb{E}\{b[T-2]\}-\min [\mathbb{E}\{b[T]\}, \mathbb{E}\{b[T-1]\}]) y[T-2] .
\end{aligned}
$$

Therefore, we can rewrite the optimization problem in (54) as

$$
\begin{aligned}
\operatorname{Min}_{y[T-2]} & (\mathbb{E}\{b[T-2]\} \\
& -\min [\mathbb{E}\{b[T]\}, \mathbb{E}\{b[T-1]\}]) y[T-2]
\end{aligned}
$$

S.t. $0 \leq y[T-2] \leq e_{1}-X-\sum_{t=1}^{T-3} y[t]$.

If $\mathbb{E}\{b[T-2]\} \leq \min [\mathbb{E}\{b[T]\}, \mathbb{E}\{b[T-1]\}]$, then

$$
y[T-2]=e_{1}-X-\sum_{t=1}^{T-3} y[t] \Rightarrow y[T-1]=y[T]=0 .
$$

Otherwise, we have $y[T-2]=0$. In that case, $y[T-1]$ and $y[T]$ are set based on a comparison between $\mathbb{E}\{b[T-1]\}$ and $\mathbb{E}\{b[T]\}$ and according to (52) and (53), where $y[T-2]=0$. Thus, any shortage of energy $e_{1}-X-\sum_{t=1}^{T-3} y[t]$ that is realized before the last three time slots will be purchased in full at the time slot with the lowest expected clearing market price. If we continue this backward induction process down to time slot $t=1$, the optimal bids will be obtained as in (3).

\section{B. Proof of Theorem 2}

Let $F(\boldsymbol{p}, \boldsymbol{x})$ denote the objective function in (5). Also let $\mathbf{1}$ denote a $T \times 1$ vector with all entries equal zero. Finally, let $\boldsymbol{p}_{-t}=p[1], \ldots, p[t-1], p[t+1], \ldots, p[T]$ denote the vector of all price bids to the day-ahead market, except for $p[t]$. We want to show that, at any time slot $t=1, \ldots, T$, we have

$$
\begin{aligned}
\underset{\boldsymbol{p}_{-t}, \boldsymbol{x}, \mathbf{1}^{T} \boldsymbol{x} \leq e_{1}}{\operatorname{Min}} F\left(p[t]>\mathbb{E}\left\{b\left[t_{b}\right]\right\}, \boldsymbol{p}_{-t}, \boldsymbol{x}\right) \\
\geq \boldsymbol{p}_{-t, \boldsymbol{x}, \mathbf{1}^{T} \boldsymbol{x} \leq e_{1}} F\left(p[t]=\mathbb{E}\left\{b\left[t_{b}\right]\right\}, \boldsymbol{p}_{-t}, \boldsymbol{x}\right)
\end{aligned}
$$

and

$$
\begin{array}{rl}
\boldsymbol{p}_{-t}, \boldsymbol{x}, \mathbf{1}^{T} \boldsymbol{x} \leq e_{1} & F\left(p[t]<\mathbb{E}\left\{b\left[t_{b}\right]\right\}, \boldsymbol{p}_{-t}, \boldsymbol{x}\right) \\
\geq & \operatorname{Min}_{\boldsymbol{p}_{-t}, \boldsymbol{x}, \mathbf{1}^{T} \boldsymbol{x} \leq e_{1}} F\left(p[t]=\mathbb{E}\left\{b\left[t_{b}\right]\right\}, \boldsymbol{p}_{-t}, \boldsymbol{x}\right) .
\end{array}
$$

Together, the above two inequalities directly result in (8).

To show (57), first, we expand the expected value terms in the objective function in (5) based on scenarios $1, \ldots, K$ with probabilities $\pi_{1}, \ldots, \pi_{K}$. Here, $K$ can be an arbitrarily large number to achieve any desirable accuracy. Let $a_{k}[1], \ldots, a_{k}[T]$ denote the realization of the day-ahead market price when scenario $k$ occurs. For any time slot $t$ and for any $p[t]>$ $\mathbb{E}\left\{b\left[t_{b}\right]\right\}$, we divide set $\mathcal{K}=\{1, \ldots, K\}$ into three disjoint subsets:

$$
\begin{aligned}
& \mathcal{K}_{1}=\left\{k \mid a_{k}[t] \leq \mathbb{E}\left\{b\left[t_{b}\right]\right\}\right\} \\
& \mathcal{K}_{2}=\left\{k \mid \mathbb{E}\left\{b\left[t_{b}\right]\right\}<a_{k}[t]<p[t]\right\}, \\
& \mathcal{K}_{3}=\left\{k \mid p[t] \leq a_{k}[t]\right\}
\end{aligned}
$$

Accordingly, we can rewrite the objective function $F(\boldsymbol{p}, \boldsymbol{x})$ as

$$
\sum_{k \in \mathcal{K}_{1}} \pi_{k} f_{k}(\boldsymbol{p}, \boldsymbol{x})+\sum_{k \in \mathcal{K}_{2}} \pi_{k} f_{k}(\boldsymbol{p}, \boldsymbol{x})+\sum_{k \in \mathcal{K}_{3}} \pi_{k} f_{k}(\boldsymbol{p}, \boldsymbol{x}),
$$

where for each $k=1, \ldots, K$, we have

$$
f_{k}(\boldsymbol{p}, \boldsymbol{x})=\sum_{\tau=1}^{T}\left(a_{k}[\tau]-\mathbb{E}\left\{b\left[t_{b}\right]\right\}\right) \mathbb{I}\left(a_{k}[\tau] \leq p[\tau]\right) x[\tau] .
$$

From (59), (61), and (63), for each $k \in \mathcal{K}_{1} \cup \mathcal{K}_{3}$, we have

$$
\begin{aligned}
& f_{k}\left(p[t]>\mathbb{E}\left\{b\left[t_{b}\right]\right\}, \boldsymbol{p}_{-t}, \boldsymbol{x}\right) \\
& =f_{k}\left(p[t]=\mathbb{E}\left\{b\left[t_{b}\right]\right\}, \boldsymbol{p}_{-t}, \boldsymbol{x}\right) .
\end{aligned}
$$

Also, from (60) and (63), for each $k \in \mathcal{K}_{2}$, we have

$$
\begin{aligned}
& f_{k}\left(p[t]>\mathbb{E}\left\{b\left[t_{b}\right]\right\}, \boldsymbol{p}_{-t}, \boldsymbol{x}\right)=\left(a_{k}[t]-\mathbb{E}\left\{b\left[t_{b}\right]\right\}\right) x[t] \\
& +\sum_{\tau=1, \tau \neq t}^{T}\left(a_{k}[\tau]-\mathbb{E}\left\{b\left[t_{b}\right]\right\}\right) \mathbb{I}\left(a_{k}[\tau] \leq p[\tau]\right) x[t]
\end{aligned}
$$


and

$$
\begin{aligned}
& f_{k}\left(p[t]=\mathbb{E}\left\{b\left[t_{b}\right]\right\}, \boldsymbol{p}_{-t}, \boldsymbol{x}\right)=0 \\
& +\sum_{\tau=1, \tau \neq t}^{T}\left(a_{k}[\tau]-\mathbb{E}\left\{b\left[t_{b}\right]\right\}\right) \mathbb{I}\left(a_{k}[\tau] \leq p[\tau]\right) x[t] .
\end{aligned}
$$

By subtracting (66) from (65), for each $k \in \mathcal{K}_{2}$, we have

$$
\begin{aligned}
& f_{k}\left(p[t]>\mathbb{E}\left\{b\left[t_{b}\right]\right\}, \boldsymbol{p}_{-t}, \boldsymbol{x}\right)-f_{k}\left(p[t]=\mathbb{E}\left\{b\left[t_{b}\right]\right\}, \boldsymbol{p}_{-t}, \boldsymbol{x}\right) \\
& \quad=\left(a_{k}[t]-\mathbb{E}\left\{b\left[t_{b}\right]\right\}\right) x[t],
\end{aligned}
$$

where

$$
a_{k}[t]-\mathbb{E}\left\{b\left[t_{b}\right]\right\}>0, \quad \forall k \in \mathcal{K}_{2} .
$$

Finally, from (62), (67), and (68), we have

$$
\begin{aligned}
& \operatorname{Min}_{\boldsymbol{p}_{-t}, \boldsymbol{x}, \mathbf{1}^{T} \boldsymbol{x} \leq e} F\left(p[t]>\mathbb{E}\left\{b\left[t_{b}\right]\right\}, \boldsymbol{p}_{-t}, \boldsymbol{x}\right) \\
& =\operatorname{Min}_{\boldsymbol{p}_{-t}, \boldsymbol{x}, \mathbf{1}^{T} \boldsymbol{x} \leq e}\left(F\left(p[t]=\mathbb{E}\left\{b\left[t_{b}\right]\right\}, \boldsymbol{p}_{-t}, \boldsymbol{x}\right)\right. \\
& +\sum_{k \in \mathcal{K}_{2}} \pi_{k} f_{k}\left(p[t]>\mathbb{E}\left\{b\left[t_{b}\right]\right\}, \boldsymbol{p}_{-t}, \boldsymbol{x}\right) \\
& \left.-\sum_{k \in \mathcal{K}_{2}} \pi_{k} f_{k}\left(p[t]=\mathbb{E}\left\{b\left[t_{b}\right]\right\}, \boldsymbol{p}_{-t}, \boldsymbol{x}\right)\right) \\
& \geq \operatorname{Min}_{\boldsymbol{p}_{-t}, \boldsymbol{x}, \mathbf{1}^{T} \boldsymbol{x} \leq e} F\left(p[t]=\mathbb{E}\left\{b\left[t_{b}\right]\right\}, \boldsymbol{p}_{-t}, \boldsymbol{x}\right) \\
& +\operatorname{Min}_{\boldsymbol{p}_{-t}, \boldsymbol{x}, \mathbf{1}^{T} \boldsymbol{x} \leq e} \sum_{k \in \mathcal{K}_{2}} \pi_{k}\left(f_{k}\left(p[t]>\mathbb{E}\left\{b\left[t_{b}\right]\right\}, \boldsymbol{p}_{-t}, \boldsymbol{x}\right)\right. \\
& \left.-f_{k}\left(p[t]=\mathbb{E}\left\{b\left[t_{b}\right]\right\}, \boldsymbol{p}_{-t}, \boldsymbol{x}\right)\right) \\
& =\operatorname{Min}_{\boldsymbol{p}_{-t}, \boldsymbol{x}, \mathbf{1}^{T} \boldsymbol{x} \leq e} F\left(p[t]=\mathbb{E}\left\{b\left[t_{b}\right]\right\}, \boldsymbol{p}_{-t}, \boldsymbol{x}\right) \\
& +\operatorname{Min}_{\boldsymbol{p}_{-t}, \boldsymbol{x}, \mathbf{1}^{T} \boldsymbol{x} \leq e} \sum_{k \in \mathcal{K}_{2}} \pi_{k}\left(a_{k}[t]-\mathbb{E}\left\{b\left[t_{b}\right]\right\}\right) x[t] \\
& =\operatorname{Min}_{\boldsymbol{p}_{-t}, \boldsymbol{x}, \mathbf{1}^{T} \boldsymbol{x} \leq e} F\left(p[t]=\mathbb{E}\left\{b\left[t_{b}\right]\right\}, \boldsymbol{p}_{-t}, \boldsymbol{x}\right),
\end{aligned}
$$

where the first equality is due to (62), the first inequality is because $\max _{x} g(x)+h(x) \leq \max _{x} g(x)+\max _{x} h(x)$, the second equality is due to (67), and the third equality is due to (68). This concludes the proof to show (57).

Next, in order to show (58), for a given time slot $t$ and $p[t]<\mathbb{E}\left\{b\left[t_{b}\right]\right\}$, we divide set $\mathcal{K}$ into three disjoint subsets

$$
\begin{aligned}
& \mathcal{K}_{1}=\left\{k \mid a_{k}[t] \leq p[t]\right\}, \\
& \mathcal{K}_{2}=\left\{k \mid p[t]<a_{k}[t]<\mathbb{E}\left\{b\left[t_{b}\right]\right\}\right\}, \\
& \mathcal{K}_{3}=\left\{k \mid \mathbb{E}\left\{b\left[t_{b}\right]\right\} \leq a_{k}[t]\right\} .
\end{aligned}
$$

We can verify that (64) still holds in this case. From (63) and (71), for any $k \in \mathcal{K}_{2}$, we have

$$
\begin{aligned}
& f_{k}\left(p[t]<\mathbb{E}\left\{b\left[t_{b}\right]\right\}, \boldsymbol{p}_{-t}, \boldsymbol{x}\right)=0 \\
& +\sum_{\tau=1, \tau \neq t}^{T}\left(a_{k}[\tau]-\mathbb{E}\left\{b\left[t_{b}\right]\right\}\right) \mathbb{I}\left(a_{k}[\tau] \leq p[\tau]\right) x[t]
\end{aligned}
$$

and

$$
\begin{aligned}
& f_{k}\left(p[t]=\mathbb{E}\left\{b\left[t_{b}\right]\right\}, \boldsymbol{p}_{-t}, \boldsymbol{x}\right)=\left(a_{k}[t]-\mathbb{E}\left\{b\left[t_{b}\right]\right\}\right) x[t] \\
& +\sum_{\tau=1, \tau \neq t}^{T}\left(a_{k}[\tau]-\mathbb{E}\left\{b\left[t_{b}\right]\right\}\right) \mathbb{I}\left(a_{k}[\tau] \leq p[\tau]\right) x[t] .
\end{aligned}
$$

By subtracting (74) from (73), for each $k \in \mathcal{K}_{2}$, we have

$$
\begin{aligned}
& f_{k}\left(p[t]<\mathbb{E}\left\{b\left[t_{b}\right]\right\}, \boldsymbol{p}_{-t}, \boldsymbol{x}\right)-f_{k}\left(p[t]=\mathbb{E}\left\{b\left[t_{b}\right]\right\}, \boldsymbol{p}_{-t}, \boldsymbol{x}\right) \\
& =-\left(a_{k}[t]-\mathbb{E}\left\{b\left[t_{b}\right]\right\}\right) x[t],
\end{aligned}
$$

where

$$
\text { - }\left(a_{k}[t]-\mathbb{E}\left\{b\left[t_{b}\right]\right\}\right)>0, \quad \forall k \in \mathcal{K}_{2} .
$$

The rest of the proof is similar to that of showing (57).

\section{Proof of Theorem 3}

Since the minimization in (10) is a linear program and all coefficients are non-positive, the objective function is minimized when $x\left[t_{a}\right]$, i.e., the variable with the lowest coefficient, is maximized. From the inequality constraint in (10), this means that at optimality, we have $x\left[t_{a}\right]=e_{1}$; and $x[t]=0$, for any $t \neq t_{a}$. For the rare scenario when not even a single price realization at any time slot in the day-ahead market may ever drop below $\mathbb{E}\left\{b\left[t_{b}\right]\right\}$, all coefficients in (10) would be zero, and at optimality, we would have $x[1]=\ldots=x[T]=0$.

\section{Proof of Theorem 4}

The minimization in (14) is a linear program. The lowest coefficient in the objective function is denoted by $\mathbb{C}\left(t_{a, \boldsymbol{p}}, p\left[t_{a, \boldsymbol{p}}\right]\right)$. If $\mathbb{C}\left(t_{a, \boldsymbol{p}}, p\left[t_{a, \boldsymbol{p}}\right]\right)>0$, then all the coefficients in the objective function in problem (14) are positive and the optimal solution is obtained as $x[0]=\ldots=x[T]$. Otherwise, we can minimize the objective function if $x\left[t_{a}\right]$, i.e., the variable with the lowest coefficient, is maximized. From the inequality constraint in (14), this means that at optimality, we must have $x\left[t_{a}\right]=e_{1}$; and $x[t]=0$, for any $t \neq t_{a}$.

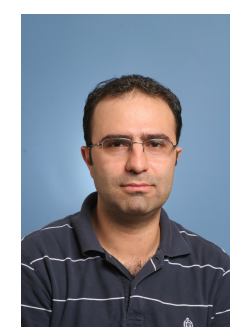

Hamed Mohsenian-Rad (S'04-M'09-SM'14) received a Ph.D. degree in Electrical and Computer Engineering from the University of British Columbia in Vancouver, Canada in 2008. Currently, he is an Assistant Professor of Electrical Engineering at the University of California at Riverside. Dr. Mohsenian-Rad is the recipient of the National Science Foundation (NSF) CAREER Award 2012, the Best Paper Award from the IEEE Power and Energy Society General Meeting 2013, and the Best Paper Award from the IEEE International Conference on Smart Grid Communications 2012. He serves as Editor for the IEEE Trans. on Smart Grid, the IEEE Communications Surveys and Tutorials, and the IEEE Communications Letters. His research interests are the design, optimization, and game-theoretic analysis of power systems and electricity market. 